\title{
APPROXIMATELY FINITE ALGEBRAS
}

BY

HAROLD WIDOM

1. Introduction. In [5, Chapter IV], a study was made of certain types of rings of operators, the approximately finite factors of type $\mathrm{I}_{1}$. These are factors of type $\mathrm{II}_{1}$ which can be suitably approximated by finite dimensional subalgebras. Two major results were obtained. Firstly it was shown that on separable Hilbert space approximately finite factors exist and are all isomorphic (Theorem XIV), and secondly a nonapproximately finite factor was produced (Theorem XVI).

In this paper we shall extend the notion of approximate finiteness to factors on nonseparable Hilbert spaces, and obtain analogs of the results of [5]. In $\$ 6$ we shall indicate how the discussion can be extended still further to finite $\mathrm{AW}^{*}$-algebras possessing a central trace.

2. Various notions of approximate finiteness. In [5, p. 759], a factor $M$ of type $\mathrm{II}_{1}$ on separable Hilbert space was defined to be approximately finite if there exists an increasing sequence $\left\{\boldsymbol{N}_{n}\right\}$ of subfactors of $\boldsymbol{M}$ of type I with $\mathrm{V} \boldsymbol{N}_{n}=\boldsymbol{M}$. (We shall use the symbol " $\mathrm{V}$ " to denote "ring generated by.") We would like to extend this definition to factors on not necessarily separable Hilbert spaces. We should not use exactly the same definition; for it is not hard to see that if $M=V N_{n}$ with $\left\{\boldsymbol{N}_{n}\right\}$ an increasing sequence of subfactors of $\boldsymbol{M}$ of type I, then $\boldsymbol{M}$ has a faithful representation as operators on separable Hilbert space, so we would actually get nothing new. However, suppose $\boldsymbol{M}=\mathrm{V} \boldsymbol{N}_{\alpha}$ where $\left\{\boldsymbol{N}_{\alpha}\right\}$ is an ascending chain of subfactors of $\boldsymbol{M}$ approximately finite in the sense of [5]. Then it is possible that $M$ does not have a faithful representation as operators'on separable Hilbert space, but we would still like to say that $\boldsymbol{M}$ is approximately finite (see [3, Theorem 2]). This leads to the following considerations.

Let $M$ be a factor of type $\mathrm{II}_{1}$ and $v$ a collection of subrings of $M$. We shall call $\mathcal{U}$ normal if for any ascending chain $\left\{N_{\alpha}\right\}$ of members of $U$ we have $V N_{\alpha} \in V$. Since the intersection of normal collections is normal, we may speak of the normal collection generated by any collection of subrings of $\boldsymbol{M}$. Let $\mathcal{U}(\boldsymbol{M})$ be the normal collection of subrings of $\boldsymbol{M}$ generated by the subfactors of type I. We shall say that $\boldsymbol{M}$ is approximately finite (A1) if $\boldsymbol{M} \in \mathcal{U}(\boldsymbol{M})$.

It follows easily from the first statement of [3, Theorem 2], that $u(M)$ must consist entirely of subfactors of $\boldsymbol{M}$. In fact, $\boldsymbol{N} \in \boldsymbol{U}(\boldsymbol{M})$ if and only if $\boldsymbol{N}$ is a subfactor of type $\mathrm{I}$ or one which is itself of type $\mathrm{II}_{1}$ and approximately

Presented to the Society, February 25, 1956; received by the editors November 23, 1955. 
finite (A1). Note that if $M$ is a factor of type $\mathrm{II}_{1}$ on separable Hilbert space, then $\boldsymbol{M}$ is approximately finite (A1) if and only if it is approximately finite in the sense of [5]. This remark also follows easily from [3, Theorem 2].

There is another way of extending the definition of approximate finiteness. Returning to the case of an approximately finite factor on separable Hilbert space, we have $M=V N_{n},\left\{N_{n}\right\}$ being an increasing sequence of subfactors of $M$ of type I. Define $P_{1}=N_{1}$ and $P_{n}=N_{n-1}^{\prime} \cap N_{n}$ for $n>1$. Then $P_{n}$ are mutually commuting subfactors of $\boldsymbol{M}$ of type I with $\boldsymbol{M}=\mathrm{V} \boldsymbol{P}_{n}$. We are thus led to the following generalization: call $\boldsymbol{M}$ approximately finite (B) if $\boldsymbol{M}=\mathrm{V} \boldsymbol{P}_{\lambda}$, the $\boldsymbol{P}_{\boldsymbol{\lambda}}$ being mutually commuting subfactors of $M$ of type I.

Finally we shall introduce another type of approximate finiteness, essentially a direct copy of Definition 4.3.1 of [5] .We shall call $M$ approximately finite (A2) if given $A_{i} \in M(i=1, \cdots, n)$ and $\epsilon>0$ we can find a subfactor $N$ of $M$ of type I containing elements $B_{i}(i=1, \cdots, n)$ such that $\left[\left[A_{i}-B_{i}\right]\right]$ $<\epsilon$. (Recall that for $A \in M,[[A]]=\operatorname{Tr}\left(A^{*} A\right)^{1 / 2}$, where $\operatorname{Tr}$ is the normalized trace on $M$.)

We shall prove below that approximate finiteness (A1) and (A2) are equivalent, and that approximate finiteness (B) is stronger than either of these $\left({ }^{1}\right)$. A factor which is approximately finite (B) is determined, up to an algebraic isomorphism, by a single cardinal number.

3. Equivalence of (A1) and (A2). Any finite factor $M$ is a metric space with metric [[ ]]. Define $\chi(\boldsymbol{M})$ to be the density character of $\boldsymbol{M}$ relative to this metric, i.e., the smallest cardinal $\aleph$ such that there exists a subset of $\boldsymbol{M}$ of cardinality $N$ which is [[ ]]-dense in $M$. Now $\boldsymbol{M}$ is a pre-Hilbert space if we define $(A, B)=\operatorname{Tr}\left(B^{*} A\right)$, so $M$ can be completed to a Hilbert space $H$. It is easily seen that if $M$ is of type II (i.e., if $H$ is infinite dimensional) then the dimension of $\boldsymbol{H}$ is just $\chi(\boldsymbol{M})$. Moreover $\boldsymbol{M}$ is faithfully represented as a ring of operators on $H[1]$. Thus, if $\chi(M)=\boldsymbol{\aleph}_{0}$ then $M$ is isomorphic to a ring of operators on separable Hilbert space.

Lemma 1. Assume $M$ is approximately finite (A2), and let $S$ be a subset of $M$ of cardinality $N$ ( $N$ infinite). Then there exists an $N \in \mathcal{N}(M)$ such that $\chi(\boldsymbol{N}) \leqq \boldsymbol{N}$ and $\mathbf{S} \subset \boldsymbol{N}$.

Proof. We shall prove the result by a transfinite induction on $\aleph$. First we prove the result for $\boldsymbol{N}=\boldsymbol{N}_{0}$. Let $S=\left\{A_{1}, A_{2}, \cdots\right\}$, and construct factors $\boldsymbol{N}_{n}$ of type I as follows. By assumption there exists a factor $\boldsymbol{N}_{1}$ of type I and $B_{11} \in N_{1}$ with $\left[\left[A_{1}-B_{11}\right]\right]<1$. Having $N_{n-1}$, apply Lemma 4.3 .5 of [5] to find a factor of type I $N_{n} \supset N_{n-1}$ such that there exist $B_{n 1}, \cdots, B_{n n} \in N_{n}$ with $\left[\left[A_{i}-B_{n i}\right]\right]<1 / n(i=1, \cdots, n)$. Let $N=V N_{n}$. Then $N \in \mathcal{U}(M)$ and $\chi(N)=N_{0}$. Now by [5, Theorem 1], $N=V N_{n}$ is the [[ ]]-closure of $U N_{n}$. But since each $A_{i}$ is in this closure, we have $A_{i} \in N$.

(1) Examples of algebras approximately finite (A) but not (B) will be exhibited in a forthcoming paper. 
Assume the result is proved for all cardinals $\left\langle\boldsymbol{N}\left(\boldsymbol{N}>\boldsymbol{N}_{0}\right)\right.$, and let $\boldsymbol{S}$ be a subset of $\boldsymbol{M}$ of cardinality $\boldsymbol{\aleph}$. If $\Omega$ is the first ordinal with cardinal $\boldsymbol{\aleph}$ we may well-order $S$ with ordinal $\Omega$. Let $A_{\alpha}(\alpha<\Omega)$ be the elements of $S$ ordered in this way. We shall construct an increasing chain $\left\{N_{\alpha}\right\}$ of members of $\mathcal{u}(M)$ such that $A_{\alpha} \in \boldsymbol{N}_{\alpha}$ and $\chi\left(\boldsymbol{N}_{\alpha}\right) \leqq \boldsymbol{N}_{0}|\alpha|$. Here $|\alpha|$ denotes the cardinal of $\alpha$. Assume we have $\boldsymbol{N}_{\alpha^{\prime}}$ for $\alpha^{\prime}<\alpha$. If $\alpha$ is finite, $V_{\alpha^{\prime}<\alpha} N_{\alpha^{\prime}}$ has a dense set $\boldsymbol{S}^{\prime}$ of cardinality $\boldsymbol{N}_{0}$. Using our induction hypothesis we can find an $\boldsymbol{N}_{\alpha} \in \mathcal{U}(\boldsymbol{M})$ such that $\boldsymbol{S}^{\prime} \cup\left\{A_{\alpha}\right\} \subset \boldsymbol{N}_{\alpha}$ and $\chi\left(\boldsymbol{N}_{\alpha}\right) \leqq \boldsymbol{N}_{0}=\boldsymbol{N}_{0}|\alpha|$. If $\alpha$ is infinite, $\bigvee_{\alpha^{\prime}<\alpha} N_{\alpha^{\prime}}$ has a dense set $\boldsymbol{S}^{\prime}$ of cardinality at most $|\alpha| \boldsymbol{\aleph}_{0}|\alpha|=|\alpha|$, and $|\alpha|<\boldsymbol{N}$. Therefore we can use the induction hypothesis to find an $\boldsymbol{N}_{\alpha} \in \mathcal{U}(\boldsymbol{M})$ such that $\boldsymbol{S}^{\prime} \cup\left\{A_{\alpha}\right\} \subset \boldsymbol{N}_{\alpha}$ and $\chi\left(\boldsymbol{N}_{\alpha}\right) \leqq|\alpha|=\boldsymbol{N}_{0}|\alpha|$. Thus in any case we can find $\boldsymbol{N}_{\alpha} \in \mathcal{U}(\boldsymbol{M})$ with $\chi\left(\boldsymbol{N}_{\alpha}\right) \leqq \boldsymbol{N}_{0}|\alpha|$, such that $\boldsymbol{S}^{\prime} \cup\left\{A_{\alpha}\right\} \subset \boldsymbol{N}_{\alpha}$, where $\boldsymbol{S}^{\prime}$ is dense in $\bigvee_{\alpha^{\prime}<\alpha} N_{\alpha^{\prime}}$. But then $N_{\alpha} \supset\left(\mathrm{V}_{\alpha^{\prime}<\alpha} N_{\alpha^{\prime}}\right) \cup\left\{A_{\alpha}\right\}$. This completes the construction of $\left\{N_{\alpha}\right\}$.

Now let $\boldsymbol{N}=\mathrm{V}_{\alpha<\Omega} \boldsymbol{N}_{\alpha}$. Then $S \subset \boldsymbol{N}$ and $\chi(\boldsymbol{N}) \leqq|\Omega| \boldsymbol{N}_{0}|\Omega|=\boldsymbol{N}$, which completes the proof.

Theorem 1. Approximate finiteness (A1) and (A2) are equivalent.

Proof. Assume $\boldsymbol{M}$ is approximately finite (A2). Then applying Lemma 1 with $\boldsymbol{S}=\boldsymbol{M}$, we can find $\boldsymbol{N} \in \mathcal{U}(\boldsymbol{M})$ such that $\boldsymbol{S} \subset \boldsymbol{N}$. But then $\boldsymbol{N}=\boldsymbol{M}$, so $\boldsymbol{M} \in \mathcal{U}(\boldsymbol{M})$ and $\boldsymbol{M}$ is approximately finite (A1).

Assume $M$ is approximately finite (A1), and let $v$ be the collection of subfactors of $M$ which are of type I or approximately finite (A2). Let $\left\{\boldsymbol{N}_{\alpha}\right\}$ be an increasing chain of members of $v, N=\mathrm{V} N_{\alpha}$. Assume $A_{1}, \cdots, A_{n} \in N$ and $\epsilon>0$ are given.

Since $N$ is the closure of $U N_{\alpha}$, we can find some $N_{\alpha} \in\left\{N_{\alpha}\right\}$ containing elements $B_{i}(i=1, \cdots, n)$ satisfying $\left[\left[A_{i}-B_{i}\right]\right]<\epsilon / 2$. Since $N_{\alpha} \in \mathcal{V}$, we can find a subfactor $P$ of $N_{\alpha}$ of type I containing elements $C_{i}(i=1, \cdots, n)$ satisfying $\left[\left[B_{i}-C_{i}\right]\right]<\epsilon / 2$. Then $P$ is a type I subfactor of $N$ containing elements $C_{i}$ satisfying $\left[\left[A_{i}-C_{i}\right]\right]<\epsilon$. Thus $N \in \mathcal{V}$. This shows that $V$ is normal. Since $v$ contains the subfactors of $\boldsymbol{M}$ of type I, we have $v \supset \mathcal{u}(\boldsymbol{M})$. Since $\boldsymbol{M}$ is approximately finite (A1), we conclude $\boldsymbol{M} \in \mathcal{V}$, so $\boldsymbol{M}$ is approximately finite (A2).

Since approximate finiteness (A1) and (A2) are equivalent, we are justified in calling either of these approximate finiteness (A). Approximate finiteness (B) implies approximate finiteness (A): Assume $\boldsymbol{M}$ is approximately finite (B). If $\boldsymbol{N}$ and $\boldsymbol{P}$ are mutually commuting subfactors of $\boldsymbol{M}$, both either of type I or approximately finite (A), then $N V P$ is either of type I or approximately finite (A). This follows easily from (A2). That $M$ is approximately finite (A) is derived from this fact using transfinite induction.

4. An isomorphism theorem. We shall prove that factors approximately finite (B) are determined, to within isomorphism, by their density characters.

LemMA 2. Let $M$ be approximately finite (B): $M=\bigvee N_{\lambda}$, the $N_{\lambda}$ being $m u$ - 
tually commuting subfactors of $M$ of type $\mathrm{I}$, but not of type $\mathrm{I}_{1}$. Then the number of $\boldsymbol{N}_{\mathrm{\lambda}}$ 's is just $\chi(\boldsymbol{M})$.

Proof. Let $R$ be the ring generated by the $N_{\lambda}$ in the purely algebraic sense. Then if $\boldsymbol{N}$ is the number of $N_{\lambda}$ 's we see that $R$ has a dense subset of cardinality $\leqq \boldsymbol{N} \cdot \boldsymbol{N}_{0}=\boldsymbol{N}$, since each $\boldsymbol{N}_{\lambda}$ has a dense subset of cardinality $\boldsymbol{N}_{0}$. Since $\boldsymbol{R}$ is dense in $\boldsymbol{M}$, we have $\chi(\boldsymbol{M}) \leqq \boldsymbol{N}$.

Form the canonical Hilbert space $H$ as in the beginning of $\S 3$. For each $\lambda$ choose an $A_{\lambda} \in N_{\lambda}$ so that $A_{\lambda}$ is not a scalar multiple of the identity. I claim that no $A_{\mu}$ is in the subspace (of $H$ ) spanned by $\left\{A_{\lambda}: \lambda \neq \mu\right\}$. For if it were, $A_{\mu}$ would be the [[ ]]-limit of a net $\left\{A_{i}\right\}$ of elements of $N_{\mu}^{\prime}$, so we would have $A_{\mu} \in N_{\mu}^{\prime}$. Thus $A_{\mu}$ would be in the center of $N_{\mu}$, contradicting our assumption that $A_{\mu}$ is not a multiple of the identity. Thus $\left\{A_{\lambda}\right\}$ is a subset of $H$ of cardinality $\aleph$ such that no $A_{\mu}$ is in the subspace spanned by the remaining $A_{\lambda}$. It follows that the dimension of $\boldsymbol{H}$ is at least $\boldsymbol{\aleph}$. Since this dimension is just $\chi(M)$, we have $\chi(M) \geqq \aleph$.

Theorem 2. Let $\boldsymbol{M}$ and $\boldsymbol{M}_{1}$ be approximately finite (B) with $\chi(\boldsymbol{M})=\chi\left(\boldsymbol{M}_{1}\right)^{\bullet}$ Then $M$ and $M_{1}$ are algebraically isomorphic.

Proof. Let $M=V N_{\lambda}, N_{\lambda}$ being mutually commuting subfactors of type I none of which, we may assume, is of type $\mathrm{I}_{1}$. Let $\left\{\lambda_{i}\right\}$ be any denumerably infinite set of $\lambda$ 's, and define $\boldsymbol{N}=\mathrm{V} \boldsymbol{N}_{\lambda_{i}}$. Then $\boldsymbol{N}$ is a subfactor of $\boldsymbol{M}$ approximately finite (B), and $\chi(\boldsymbol{N})=\boldsymbol{N}_{0}$. Thus $\boldsymbol{N}$ is an approximately finite factor on separable Hilbert space. By [5, Lemma 4.4.3], we can find an increasing sequence $\left\{\boldsymbol{P}_{n}\right\}$ of subfactors of $\boldsymbol{N}$ such that $\boldsymbol{P}_{n}$ is of type $\mathrm{I}\left(2^{n}\right)$ and $\mathrm{V} \boldsymbol{P}_{n}=\boldsymbol{N}$. Let $\boldsymbol{R}_{1}=P_{1}$ and $\boldsymbol{R}_{n}=P_{n-1}^{\prime} \cap P_{n}$ for $n>1$. Then $\boldsymbol{R}_{n}$ are mutually commuting subfactors of $N$ of type $\mathrm{I}_{2}$ with $N=\mathrm{V} \boldsymbol{R}_{n}$.

By Lemma 2 the set $\{\lambda\}$ has cardinality $\aleph=\chi(M)$, so we may split it into $\boldsymbol{N}$ mutually disjoint subsets of cardinality $\boldsymbol{\aleph}_{0}$. To each of these subsets apply the above construction to get factors of type $\mathrm{I}_{2}$. Changing notation, we now have $M=V N_{\lambda}$, where $N_{\lambda}$ are mutually commuting subfactors of type $I_{2}$. Now we can apply the same process to $\boldsymbol{M}_{1}$ to get $\boldsymbol{M}_{1}=\mathrm{V} \boldsymbol{P}_{\lambda}$, where $\boldsymbol{P}_{\lambda}$ are mutually commuting subfactors of $\boldsymbol{M}_{1}$. We may use the same indexing set $\{\lambda\}$ in both cases since there are just $\aleph$ subfactors in both cases.

Since $\boldsymbol{N}_{\lambda}$ and $\boldsymbol{P}_{\lambda}$ are both factors of type $\mathrm{I}_{2}$ we can find an isomorphism onto $\phi: N_{\lambda} \rightarrow P_{\lambda}$. We use the symbol " $\phi$ " for all the $\lambda$ 's since the intersection of any two $N_{\lambda}$ 's is just the complex numbers, where all the $\phi$ 's agree. We shall extend the various $\phi$ 's to a single isomorphism between $\boldsymbol{M}$ and $\boldsymbol{M}_{1}$. Let $\boldsymbol{N}$ and $P$ be the rings generated (in the purely algebraic sense) by $\left\{N_{\lambda}\right\}$ and $\left\{P_{\lambda}\right\}$ respectively. Now any $A \in N$ has the form $A=\sum_{i=1}^{k} A_{\imath}^{1} \cdots A_{\imath}^{n}$ with $A_{i}^{j} \in N_{\lambda_{j}}$. For such an $A$ define

$$
\psi(A)=\sum_{i=1}^{k} \phi\left(A_{i}^{1}\right) \cdots \phi\left(A_{i}^{n}\right) \in P .
$$


Then $\psi$ is just the tensor product of the various $\phi$ 's. It follows easily from the uniqueness of the trace that $\psi$ is a trace-preserving isomorphism. Since $\boldsymbol{N}$ is dense in $M$ and $P$ is dense in $M_{1}, \psi$ can be extended to an isomorphism between $M$ and $M_{1}$.

5. Examples. In [5, Theorem XIII], a nonconstructive proof was given of the existence of approximately finite factors. We can given an equally nonconstructive proof for the existence of factors approximately finite (A).

THEOREM 3. Any factor of type $\mathrm{II}_{1}$ contains a maximal subfactor approximately finite (A).

Proof. Let $M$ be the given factor of type $\mathrm{II}_{1}$, and consider the collection $\mathfrak{u}(\boldsymbol{M})$. By the very definition of $\mathfrak{u}(\boldsymbol{M})$, we can apply Zorn's lemma to find an $N \in \mathcal{U}(\boldsymbol{M})$ maximal relative to the partial order of inclusion. Since $\boldsymbol{N} \in \mathcal{U}(\boldsymbol{N})$, we shall be through if we can show $\boldsymbol{N}$ is of type II. But if $\boldsymbol{N}$ is of type I we can apply Lemma 4.4.2 of [5] to obtain a member of $\mathfrak{u}(\boldsymbol{M})$ properly containing $\boldsymbol{N}$. Thus $\boldsymbol{N}$ is of type II.

By the method of [5, Chapter V], it is easy to construct factors approximately finite (B). Let $G$ be a countable group satisfying the conditions of Lemma 5.2.2 and 5.3.4 of [5]. For any infinite cardinal $\aleph$, let $G_{0}$ be the weak direct product of $\aleph$ copies of $G$, and let $I_{0}^{\prime}$ be the factor obtained from $G_{0}$ by the method of $\$ 5.3$ of [5]. Corresponding to the decomposition of $G_{0}$ into mutually commuting subgroups isomorphic to $G$, there is a decomposition of $I_{0}^{\prime}$ into mutually commuting subfactors isomorphic to $I^{\prime}$ (where $I^{\prime}$ is the factor obtained from $G$ ). Since $I^{\prime}$ is approximately finite with density character $\aleph_{0}, I_{0}^{\prime}$ is approximately finite (B) with density character $\aleph$. We have proved,

ThEOREM 4. Given any infinite cardinal $\mathfrak{N}$, there exists a factor $M$ approximately finite (B) with $\chi(\boldsymbol{M})=\boldsymbol{N}$.

Nonapproximately finite factors were produced in [5, Chapter IV]. Using these factors, we can produce factors of type $\mathrm{II}_{1}$ with arbitrary density characters which are not approximately finite. Rather than go through this, however, we shall show that certain other factors of type $\mathrm{II}_{1}$, which arise in a very natural way, are not approximately finite.

We shall be concerned with certain factors considered by Wright [8]. Let .$N$ be a finite $A W^{*}$-algebra of type I (see $[4$, p. 236]), with homogeneous direct summands $N_{n}, N_{n}$ being of type I $\left(p_{n}\right)$ with $p_{1}<p_{2}<\ldots$ If the maximal ideal space of the center of $N_{n}$ is $X_{n}$, then the maximal ideal space $X$ of the center of $\boldsymbol{N}$ can be identified with the Stone-Cech compactification of the disjoint union $Y=U X_{n}$. Now every $t \in X$ defines a maximal ideal $\boldsymbol{M}_{t}$ of $\boldsymbol{N}: \boldsymbol{M}_{\boldsymbol{t}}$ $=\left[A \in N: \operatorname{Tr}\left(A^{*} A\right)(t)=0\right]$, where $\operatorname{Tr}$ is the central trace on $N$. The theorem of Wright that will concern us is [8, Theorem 5.1], namely that $t \in X-Y$ implies that $N / M_{t}$ is an $\mathrm{AW}^{*}$ factor of type $\mathrm{II}_{1}$ with trace. It is shown in [1] 
that $\boldsymbol{N} / \boldsymbol{M}_{\boldsymbol{t}}$ is then a $\mathrm{W}^{*}$ algebra, i.e., it has a faithful representation as a ring of operators. Moreover it is shown in [2] that any faithful representation of $\boldsymbol{N} / \boldsymbol{M}_{\boldsymbol{t}}$ as a ring of operators must be on a nonseparable Hilbert space. We shall show here that $\boldsymbol{N} / \boldsymbol{M}_{\boldsymbol{t}}$ is not approximately finite (A).

First we prove a few lemmas. We shall write $M=N / M_{t}$. The first lemma shows that a type $\mathrm{I}_{p}$ subfactor of $M$ can almost be lifted to a type $\mathrm{I}_{p}$ subalgebra of $\boldsymbol{N}$. We shall denote the center of $\boldsymbol{N}_{n}$ by $\boldsymbol{Z}_{n}$, and for the given integer $p, N_{p}=\left[n: p\right.$ divides $\left.p_{n}\right]$. Note that $N$ is the direct sum of the $\boldsymbol{N}_{n}$, so any element of $N$ has the form $\left\{A_{n}\right\}$ with $A_{n} \in N_{n}$.

Lemma 3. Let $P$ be a subfactor of $M$ of type $I_{p}$, and let $\epsilon>0$ be given. Then we can find a unitary $\bar{U} \in M$ with $[[\bar{U}-I]]<\epsilon$, and for each $n \in N_{p}$ a subalgebra $P_{n}$ of $N_{n}$ of type $\mathrm{I}_{p}$ with center $Z_{n}$, such that each $\bar{A} \in \bar{U} P \bar{U}^{*}$ can be lifted to an element of the form $\left\{A_{n}\right\} \in N$ with $A_{n} \in P_{n}$ whenever $n \in N_{p}$.

Proof. For each $n$, find a projection $E_{n} \in N_{n}$ with $D\left(E_{n}\right)=\left[p_{n} / p\right] p / p_{n}$, where $D$ is the normalized dimension function from the projections of $N_{n}$ to $Z_{n}$; let $\boldsymbol{N}^{\prime}=\sum E_{n} N_{n} E_{n}$, the direct sum of the $E_{n} N_{n} E_{n}$. Since $D\left(E_{n}\right) \rightarrow 1$ (note that $p_{n} \rightarrow \infty$ ), every element of $M$ can be lifted to one of $\boldsymbol{N}^{\prime}$, so $\boldsymbol{M}=\boldsymbol{N}^{\prime} / \boldsymbol{M}_{\boldsymbol{t}} \cap \boldsymbol{N}^{\prime}$.

Since, except for the finitely many $n$ such that $p_{n}<p, E_{n} N_{n} E_{n}$ contains a set of $p$ mutually equivalent orthogonal projections with supremum $E_{n}$, we can find a subalgebra $Q_{n}$ of $E_{n} N_{n} E_{n}$ of type $I_{p}$ with center $E_{n} Z_{n}$; for $p_{n}<p$ set $Q_{n}=\{0\}$. By [8, Theorem 5.1], $\sum Q_{n} / M_{t} \cap \sum Q_{n}$ is a subfactor of $M$ of type $\mathrm{I}_{p}$. Since any two subfactors of $M$ of type $\mathrm{I}_{p}$ are unitarily equivalent, we can find a unitary $\bar{V} \in M$ such that $P=\bar{V}\left(\sum \boldsymbol{Q}_{n} / \boldsymbol{M}_{t} \cap \sum \boldsymbol{Q}_{n}\right) \bar{V}^{*}$. By the spectral theorem we can find a unitary $\bar{W} \in M$ with $\|\bar{W}-\bar{V}\|<\epsilon$ (and so certainly with $[[\bar{W}-\bar{V}]]<\epsilon$ ) such that the spectrum of $\bar{W}$ is not the entire unit circle.

I claim that $\bar{W}$ can be lifted to a unitary of $N^{\prime}$. In fact, if $z$ is not in the spectrum of $\bar{W}$ then $\bar{A}=-i(\bar{W}+z)(\bar{W}-z)^{-1}$ is a self-adjoint element of $M$, and so can be lifted to a self-adjoint element $\left\{A_{n}\right\}$ of $N^{\prime}$. Then if $W_{n}$ $=z\left(A_{n}-i\right)\left(A_{n}+i\right)^{-1}$, the element $\left\{W_{n}\right\}$ of $N^{\prime}$ is unitary and maps into $\bar{W}$ under the natural map $\boldsymbol{N}^{\prime} \rightarrow M$.

Let $\bar{U}=\bar{W} \bar{V}^{*}, P_{n}=W_{n} \boldsymbol{Q}_{n} W_{n}^{*}$. Then $\bar{U}$ is unitary with $[[\bar{U}-I]]<\epsilon$. Also, for $n \in N_{p}$ we have $E_{n}=I$, so $P_{n}$ is of type $I_{p}$ with center $Z_{n}$ for such $n$. Now $\bar{U} \cdot P \bar{U}^{*}=\bar{W}\left(\sum \boldsymbol{Q}_{n} / M_{t} \cap \sum \boldsymbol{Q}_{n}\right) \bar{W}^{*}=\sum P_{n} / M_{t} \cap \sum P_{n}$. The result is therefore established.

The above lemma might be vacuous: it is possible that no $p_{n}$ is divisible by $p$. The following lemma shows that this situation can be circumvented.

Lemмa 4. We can find for each $n$ a subalgebra $N_{n}^{\prime}$ of $N_{n}$ such that,

(1) $N_{n}^{\prime}$ is of type $\mathrm{I}\left(q_{n}\right)$, where $q_{n}$ is divisible by $n$,

(2) the algebras $M$ and $\sum N_{n}^{\prime} / M_{t} \cap \sum N_{n}^{\prime}$ are isomorphic. 
Proof. Let $q_{n}=n\left[p_{n} / n\right]$; find a projection $E_{n} \in N_{n}$ with $D\left(E_{n}\right)=q_{n} / p_{n}$ and set $N_{n}^{\prime}=E_{n} N_{n} E_{n}$. Then $N_{n}^{\prime}$ is of type $\mathrm{I}\left(q_{n}\right)$ with center $E_{n} Z_{n}$ and (1) is immediate.

There is a natural *-homomorphism $\phi: \sum N_{n}^{\prime} \rightarrow M$. We shall show that $\phi$ is onto and has kernel $M_{t} \cap \sum N_{n}^{\prime}$, which will complete the proof. That $\phi$ is onto is the assertion that every element of $M$ can be lifted to one of $\sum N_{n}^{\prime}$, and this follows from the fact that $D\left(E_{n}\right) \rightarrow 1$. That the kernel of $\phi$ is $M_{t} \cap \sum N_{n}^{\prime}$ is immediate.

The next lemma, the really crucial one, follows immediately from the main result of [6] and the fact that an algebra of type $I_{p}$ is just the $p \times p$ matrix algebra over the center of the original algebra.

Lemma 5. There exists an $\epsilon>0$ with the following property: for any $m>0$ and $p>1$, and algebra $N$ of type $\mathrm{I}_{p m}$ with center $Z$, there exists an $A \in N$ with $\|A\| \leqq 1$ such that if $\boldsymbol{N}^{\prime}$ is a type $\mathrm{I}_{m}$ subalgebra of $\boldsymbol{N}$ with center $\boldsymbol{Z}$ then $[[B-A]]>\epsilon$ for all $B \in N^{\prime}$.

THEOREM 5. The algebra $M$ is not approximately finite (A).

Proof. By Lemma 4 we may assume each $p_{n}$ is divisible by $n$; we shall begin with $n=2$. Apply Lemma 5 to each $N_{n !}$, with $p=n$ and $m=(n-1)$ !, i.e. find $A_{n !} \in N_{n !}$ with $\left\|A_{n !}\right\| \leqq 1$ such that if $N^{\prime}$ is a subalgebra of type $\mathrm{I}_{p}$ of $N_{n !}$ with center $Z_{n}$, and $p \leqq n-1$, then $\left[\left[B-A_{n !}\right]\right]>\epsilon$ for all $B \in N^{\prime}$.

If $k$ is not of the form $n$ !, define $A_{k}=0$. Let $\bar{A}$ be the element of $M$ corresponding to $\left\{A_{n}\right\} \in N$. We shall show that no type I subfactor of $M$ can contain an element approximating $\bar{A}$ to within $\epsilon / 2$. In fact, let $P$ be a type I subfactor of $M$, say of type $\mathrm{I}_{p}$, and let $\bar{B} \in P$. We may apply Lemma 3 to find a unitary $\bar{U} \in M$ with $[[\bar{U}-I]]<\left.\epsilon\|\bar{B}\|\right|^{-1} / 4$ and a subalgebra $P_{n}$ of $N_{n}$ with the stated properties. Note that $N_{p}$ contains all integers of the form $k$ ! with $k \geqq p$. Thus $\bar{U} \bar{B} \bar{U}^{*}$ can be lifted to an element $\left\{B_{n}\right\}$ with $B_{n} \in \boldsymbol{P}_{n}$ for $n$ of the form $k !(k \geqq p)$. But we know that for $n=k$ ! with $k>p$ we have $\left[\left[B_{n}-A_{n}\right]\right]>\epsilon$. Thus $\left[\left[B_{n}-A_{n}\right]\right]>\epsilon$ for infinitely many $n$, so $\left[\left[\bar{U} \bar{B} \bar{U}^{*}-\bar{A}\right]\right]>\epsilon$. Now $\left[\left[\bar{B}-\bar{U} \bar{B} \bar{U}^{*}\right]\right] \leqq 2\|B\|[[\bar{U}-I]]<\epsilon / 2$. Therefore $[[\bar{B}-\bar{A}]]>\epsilon / 2$. Since this holds for every element $\bar{B}$ of every type I subfactor of $M, M$ cannot be approximately finite (A).

6. Approximately finite $\mathbf{A W}^{*}$-algebras. A study of the work above will reveal the fact that in studying approximately finite factors no use whatever was made of the underlying Hilbert space; the theory is entirely nonspatial. We could just as well have worked with a finite $\mathrm{AW}^{*}$-factor with trace. (For the basic theory of $\mathrm{AW}^{*}$-algebras we refer the reader to [4].) It happens that such an algebra has a faithful representation as a ring of operators on some Hilbert space [1], but this Hilbert space is not necessary for considerations of approximate finiteness. This suggests that the theory of approximately finite factors can be extended to arbitrary $A W^{*}$-algebras of type $I_{1}$ with 
central trace - the generalization here being from the factor case to the case of general center. It turns out that this extension can be made, and that the results obtained parallel almost completely those obtained above. In this section we shall merely state the results in the more general situation.

We start with an $\mathrm{AW}^{*}$-algebra $\boldsymbol{M}$ of type $\mathrm{II}_{1}$ with center $\boldsymbol{Z}, \boldsymbol{M}$ possessing a central trace $\operatorname{Tr}$. For $A \in M,[[A]]=\operatorname{Tr}\left(A^{*} A\right)^{1 / 2}$. We define "normal collection" as before, except that now the symbol " $\mathrm{V}$ " means "AW-subalgebra generated by;" and $u(\boldsymbol{M})$ is the normal collection of AW*-subalgebras of $\boldsymbol{M}$ generated by the $A W^{*}$-subalgebras of type I with center $\boldsymbol{Z}$. The definitions of approximately finiteness now read:

(A1) $\boldsymbol{M} \in \mathcal{u}(\boldsymbol{M})$,

(A2) given $A_{1}, \cdots, A_{n} \in M$ and $\epsilon>0$ we can find an AW*-subalgebra $N$ of $\boldsymbol{M}$ of type $\mathrm{I}$ with center $\boldsymbol{Z}$ containing elements $B_{i}$ such that $\left[\left[A_{i}-B_{i}\right]\right]$ $\leqq \epsilon(i=1, \cdots, n)$,

(B) We can find $\boldsymbol{P}_{\lambda}$, mutually commuting $\mathrm{AW}^{*}$-subalgebras of $\boldsymbol{M}$ of type I with center $\boldsymbol{Z}$, such that $\boldsymbol{M}=\mathrm{V} \boldsymbol{P}_{\boldsymbol{\lambda}}$.

We define $\chi_{1}(\boldsymbol{M})$ to be the smallest cardinal $\aleph$ such that there exists a subset $\boldsymbol{S}$ of $\boldsymbol{M}$ of cardinality $\boldsymbol{\aleph}$ with $\boldsymbol{S} \vee \boldsymbol{Z}=\boldsymbol{M}$. It is seen that in the factor case, $\chi_{1}(M)$ need not equal the $\chi(M)$ defined in $\S 3$. In this case the inequalities $\chi_{1}(\boldsymbol{M}) \leqq \chi(M) \leqq \boldsymbol{N}_{0} \cdot \chi_{1}(\boldsymbol{M})$ are easily seen to hold.

The analogue of Theorem 1 is,

Theorem 6. Approximate finiteness (A1) and (A2) are equivalent.

As before we call an algebra approximately finite (A) if either (A1) or (A2) holds. We have,

THEOREM 7. Approximate finiteness (B) implies approximate finiteness (A). If $\boldsymbol{M}$ is approximately finite (A) and $\chi_{1}(\boldsymbol{M}) \leqq \aleph_{0}$ then $\boldsymbol{M}$ is approximately finite (B).

Using the central trace on $\boldsymbol{M}$, we can construct an $\mathrm{AW}^{*}$-module over $\boldsymbol{Z}$ in a natural way. For $A, B \in M$ define $(A, B)=\operatorname{Tr}\left(B^{*} A\right)$. Then $M$ is an inner product space over $\boldsymbol{Z}$ (see $[7, \S 2]$ ). By forming the $\mathrm{AW}^{*}$-completion $\boldsymbol{H}(\boldsymbol{M})$ of $\boldsymbol{M}$ we obtain an $\mathrm{AW}^{*}$-module over $\boldsymbol{Z}$. The analogue of Theorem 2 is,

Theorem 8. Let $\boldsymbol{M}$ and $\boldsymbol{M}^{\prime}$ be approximately finite (B) with centers $\boldsymbol{Z}$ and $\boldsymbol{Z}^{\prime}$ respectively. Assume $\boldsymbol{Z}$ is locally countably decomposable. Then $\boldsymbol{M}$ and $\boldsymbol{M}^{\prime}$ are isomorphic if and only if $\boldsymbol{H}(\boldsymbol{M})$ and $\boldsymbol{H}\left(\boldsymbol{M}^{\prime}\right)$ are isomorphic. If $\boldsymbol{H}(\boldsymbol{M})$ and $\boldsymbol{H}\left(\boldsymbol{M}^{\prime}\right)$ are isomorphic, and $\boldsymbol{\phi}$ is the isomorphism between $\boldsymbol{Z}$ and $\boldsymbol{Z}^{\prime}$ inherent in the isomorphism between $\boldsymbol{H}(\boldsymbol{M})$ and $\boldsymbol{H}\left(\boldsymbol{M}^{\prime}\right)$, the isomorphism between $\boldsymbol{M}$ and $\boldsymbol{M}^{\prime}$ can be chosen to be an extension of $\phi$.

Theorem 3 goes over almost verbatim.

Theorem 9. Any $A W^{*}$-algebra of type $\mathrm{II}_{1}$ with center $\boldsymbol{Z}$ which possesses a 
central trace contains a maximal $A W^{*}$-subalgebra with center $\boldsymbol{Z}$ which is approximately finite (A).

In the factor case we saw that an algebra approximately finite (B) is determined by an infinite cardinal number, and furthermore that there is such an algebra for each cardinal number (Theorems 2 and 4). In the case of general (or almost general) center an algebra approximately finite (B) is determined by an $\mathrm{AW}^{*}$-module (Theorem 8 ). Not every $\mathrm{AW}^{*}$-module can arise in this way. Call an $\mathrm{AW}^{*}$-module $\boldsymbol{H}$ purely infinite if the algebra of operators on $\boldsymbol{H}$ is purely infinite $\left[4\right.$, p. 240]. It is seen that the $\mathrm{AW}^{*}$-modules $\boldsymbol{H}(\boldsymbol{M})$ are always purely infinite.

THEOREM 10. Let $\boldsymbol{H}$ be a purely infinite $\mathrm{AW}^{*}$-module over the commutative $A W^{*}$-algebra $\boldsymbol{Z}$. Then there exists an $A W^{*}$-algebra $\boldsymbol{M}$ with center $\boldsymbol{Z}$ which is approximately finite (B) and such that $\boldsymbol{H}(\boldsymbol{M})$ is isomorphic to $\boldsymbol{H}$.

\section{BIBLIOGRAPHY}

1. J. Feldman, Embedding of $A W^{*}$ algebras, Duke Math. J. vol. 23 (1956) pp. 303-307.

2. - Nonseparability of certain finite factors, Proc. Amer. Math. Soc. vol. 7 (1956) pp. 23-26.

3. B. Fuglede and R. V. Kadison, On a conjecture of Murray and von Neumann, Proc. Nat. Acad. Sci. U.S.A. vol. 37 (1951) pp. 420-425.

4. I. Kaplansky, Projections in Banach algebras, Ann. of Math. vol. 53 (1951) pp. 235249.

5. F. J. Murray and J. von Neumann, On rings of operators IV, Ann. of Math. vol. 44 (1943) pp. 716-808.

6. J. von Neumann, Approximative properties of matrices of high order, Portugaliae Mathematica vol. 3 (1942) pp. 1-62.

7. H. Widom, Embedding in algebras of type I, Duke Math. J. vol. 23 (1956) pp. 309-324.

8. F. B. Wright, A reduction for algebras of finite type, Ann. of Math. vol. 60 (1954) pp. 560570.

University of Chicago,

Chicago, Ill.

Cornell University,

ITHACA, N. Y. 